\title{
Using CFD in the Design Process of a Toilet Bowl
}

\author{
António Gameiro Lopes ${ }^{1}$, Vítor Costa ${ }^{2}$ \\ ${ }^{1}$ ADAI-LAETA, Departamento de Engenharia Mecânica \\ Universidade de Coimbra, 3030-788 Coimbra, Portugal \\ antonio.gameiro@dem.uc.pt; v.costa@ua.pt \\ ${ }^{2}$ Centro de Tecnologia Mecânica e Automação, Departamento de Engenharia Mecânica \\ Universidade de Aveiro, Campus Universitário de Santiago, 3810-193 Aveiro, Portugal
}

\begin{abstract}
In this work, a water flow analysis study is performed, leading to a toilet bowl satisfying, simultaneously, functionality, design and present standard requirements. An existing toilet bowl is used as the starting point, which is experimentally tested for discharge analysis and flow visualization. The numerical simulation of the water discharge in the existing bowl is conducted using the software package ANSYS-CFX, and the results are compared with the experimental data. This workflow allowed to tune the parameters and options to be considered in the numerical simulations. Comparisons and validation include mainly the gravity induced water mass flow rate, the water flow spreading and action over the toilet bowl inner surface, and the non-spreading of the discharged water out of the top of the toilet bowl. Once confident with the numerical simulation results, different designs of the toilet bowl and different discharge conditions can be tested in what concerns the aforementioned requisites - this a much easier, less expensive, and quick process than a trial-and-error approach using prototype toilet bowl pieces and experimental analysis.
\end{abstract}

Keywords: Toilet Bowl Design, Numerical Simulation, Water Flow Discharge, Validation, New Designs.

\section{Introduction}

Home elements have increasing requirements in many aspects, like resources (water, energy or other) consumption, functionality, user-friendliness, compactness, and design. These increasing requirements are also associated with shortened lifetimes of the home elements, thus frequently requiring new solutions to the market in short time, with affordable processes. CFD can help especially when fluid flows are present, reducing time and costs of the design and development phases, even if requiring more specialized knowledge. If this is true in general, it is also the case when the toilet bowl is the home element under consideration.

Increasing design and compactness requirements, combined with water consumption reductions and increasingly demanding standard requirements, need to be considered when proposing new toilet bowls to the market. Considering the European manufacturers and market, the present relevant Standards are the NF EN 33 [1] and the NF EN 997 [2]. This is thus an area, or product, where CFD can help towards faster, cheaper, different and better solutions. No similar studies were found in the literature, and only some, few, simple and short, visualization movies and examples from software companies were found, such as [3] and [4].

The approach followed in this work includes the experimental flow visualization testing of an existing toilet bowl during the discharge process, and its corresponding numerical simulation. Main parameters observed from both the experimental and numerical results are the water mass flow rate, the water flow action and spreading over the toilet bowl inner surface (for cleaning action and dejects transportation), and the non-spreading of the discharged water out of the top of the toilet bowl (which could occur for high water discharge mass flow rates if the bowl design is inappropriate). Water flow visualization and video recording are used for the observation of the water flow spreading over the toilet bowl inner surface, and of the non-spreading of the discharged water out of the top of the toilet bowl.

Experimental and numerical results are compared, and the parameters considered for the numerical simulations adjusted if/when needed in order to approximate the numerical results to the experimental ones. This is thus a phase of analysis, tuning and validation of the numerical simulations.

Once confidence achieved on the numerical setup, this approach will allow the study for different toilet bowl geometries and designs, as well as different discharge conditions. 


\section{Experimental}

The toilet bowl considered in the first phase of this study is the SunLife model from Sanindusa, which is shown in Fig. 1. The discharge is made using a standard installation, where an elevated cistern provides a gravity-driven water discharge flow. Visualization of the water flow spreading over the toilet bowl inner surface is made considering three different level/contour lines registered in the inner surface of the toilet bowl. Water discharged into the bowl is pink coloured to obtain a higher contrast with the white colour of the inner bowl surface. The discharge process and the water flow in the toilet bowl are recorded for post-processing and analysis. The discharge flow rate was measured by monitoring the hydrostatic pressure at the bottom of the water reservoir. Results, as depicted in Fig. 2, show an initial flow acceleration as a consequence of the inertia associated to the water in the cistern, followed by a temporal region where the flow rate gradually decreases. The process ends with a strong deceleration, after which the reservoir is empty. Simulations were performed for a water flow rate $0.85 \mathrm{~L} / \mathrm{s}$, which corresponds to the initial stages after the flow acceleration.

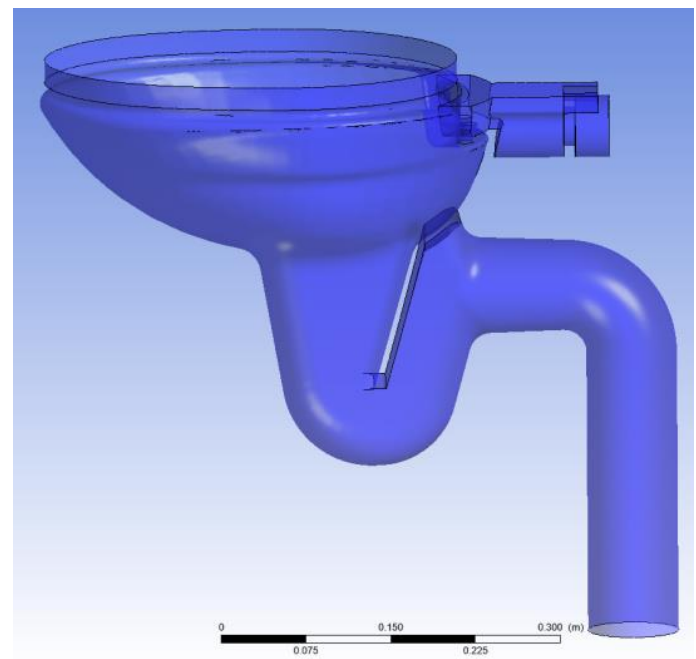

Fig. 1: The SunLife toilet bowl model from Sanindusa.

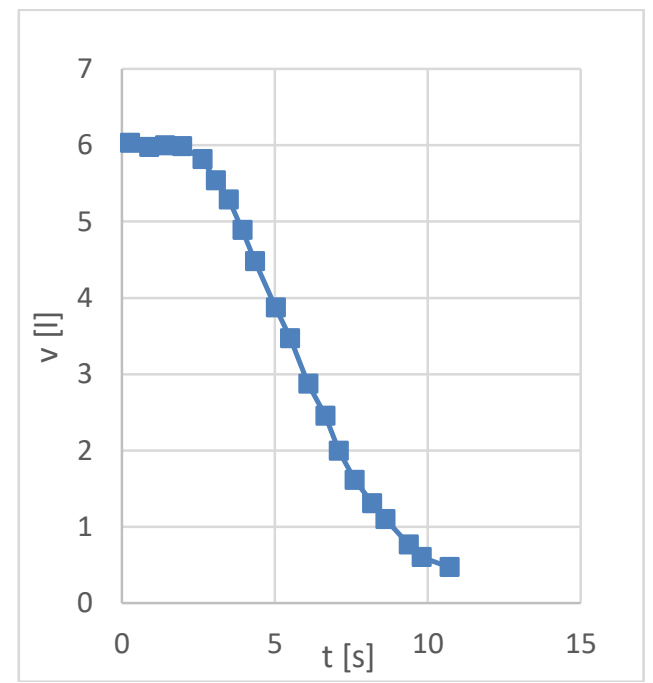

Fig. 2: Water volume in the cistern as a function of time.

\section{Numerical Simulation}

The numerical simulation was conducted in ANSYS CFX [5], using a mesh of unstructured type. An inflation layer with a total thickness of $2 \mathrm{~cm}$ and first layer thickness of $0.2 \mathrm{~mm}$ was adopted near solid boundaries, in order to provide 
better resolution for the velocity gradients at those regions (Fig. 3). This inflation layer also allowed a better capture of the steep gradients in the air-water interface. After preliminary mesh-dependency tests, the final mesh size was set at $3 \mathrm{~mm}$ for surface mesh and $5 \mathrm{~mm}$ for volume mesh, resulting in a total of 1.5 million nodes and 4 million elements.

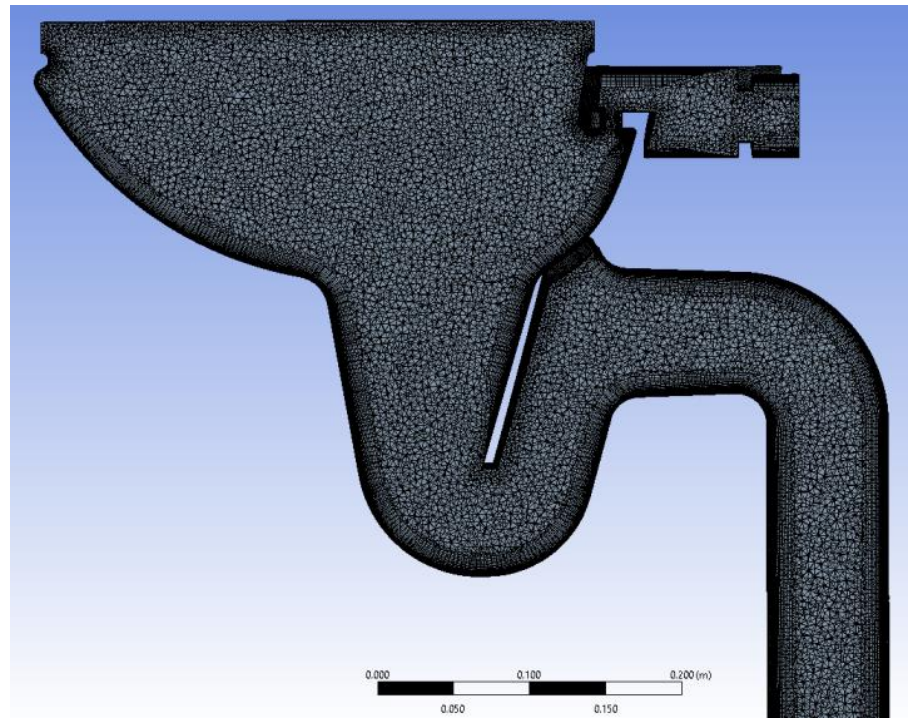

Fig. 3: Mesh on the sanitary bowl central plane.

The free surface flow calculation was performed using the homogeneous model, where all fluids share the same velocity and turbulence fields at the water-air interface. The air-water interaction was modelled using a surface tension coefficient of $0.0728 \mathrm{~N} / \mathrm{m}$. The k- $\varepsilon$ turbulence model was adopted to take into account the effects of velocity fluctuations upon the mean flow fields. At the inlet, a turbulence intensity of $5 \%$ was assigned.

This is a complex problem of gravity-driven fluid flow, with the water entering the bowl at several locations and producing a complex pattern of free-surface interaction. Although a constant flow rate was imposed to compare simulations with observations at a specific stage of the discharge process, unsteadiness associated with the complex interactions between both phases had to be taken into account. Accordingly, a transient approach was adopted. To reduce temporal discretization errors, a maximum Courant number of 1 was ensured during all the numerical simulation.

\section{Results and Validation}

Figure 4 represents the flow in the bowl, including the inlet and outlet locations. This simulation result shows three central discharge holes with the purpose of increasing the outlet flow speed. A lateral hole produces a swirling flow for a more uniform water coverage of the bowl inner surface.

Comparison of the experimental visualization of the water flow in the toilet bowl during the discharge phase with the corresponding numerical results is presented in Fig. 5, for the instant $2.5 \mathrm{~s}$ after the beginning of the discharge process. This figure represents a top view of the bowl. A good agreement between results obtained from both approaches is observed, the water action and spreading over the inner bowl surface obtained numerically being very close to that obtained experimentally. Some small differences in the level lines are apparent in the real and in the virtual bowl, mainly due to the essentially manual process of surface finishing of the toilet bowls, for which repeatability does not exist in a strict sense. One may also notice some water flow mismatch below the lateral hole, with the simulation showing a region with little water coverage. In the experiment, the water presents a wider spread. The reason for this discrepancy is believed to be related with differences between the inlet surface in the CAD models and in the real prototype.

The flow unsteadiness may be appreciated in Fig. 6, where the water free surface is represented for a temporal sequence with a time step of $0.1 \mathrm{~s}$. 


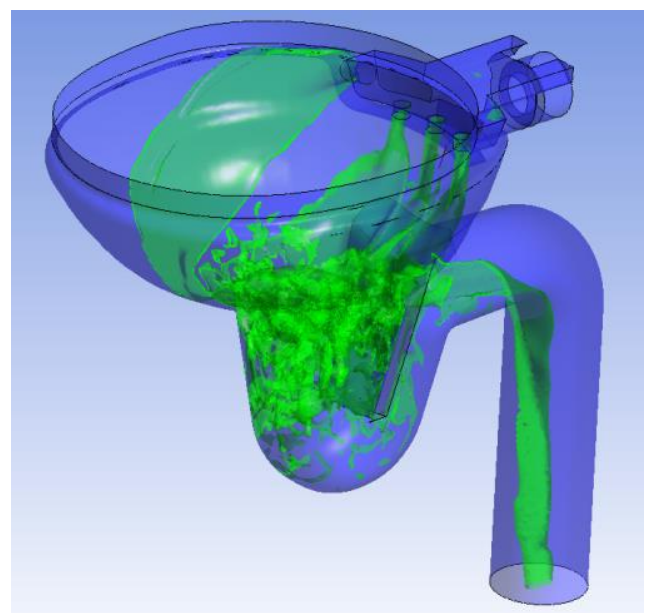

Fig. 4: Water flow in the bowl, including the inlet and outlet locations.
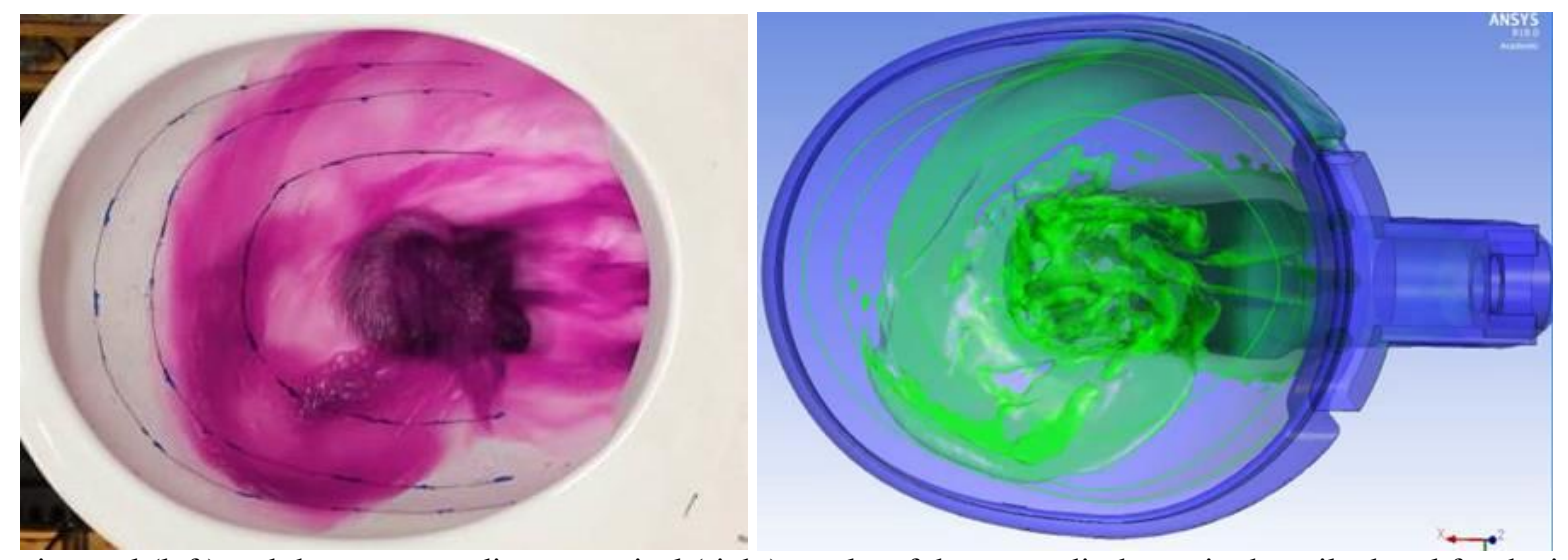

Fig. 5: Experimental (left) and the corresponding numerical (right) results of the water discharge in the toilet bowl for the instant $2.5 \mathrm{~s}$ after the discharge beginning.
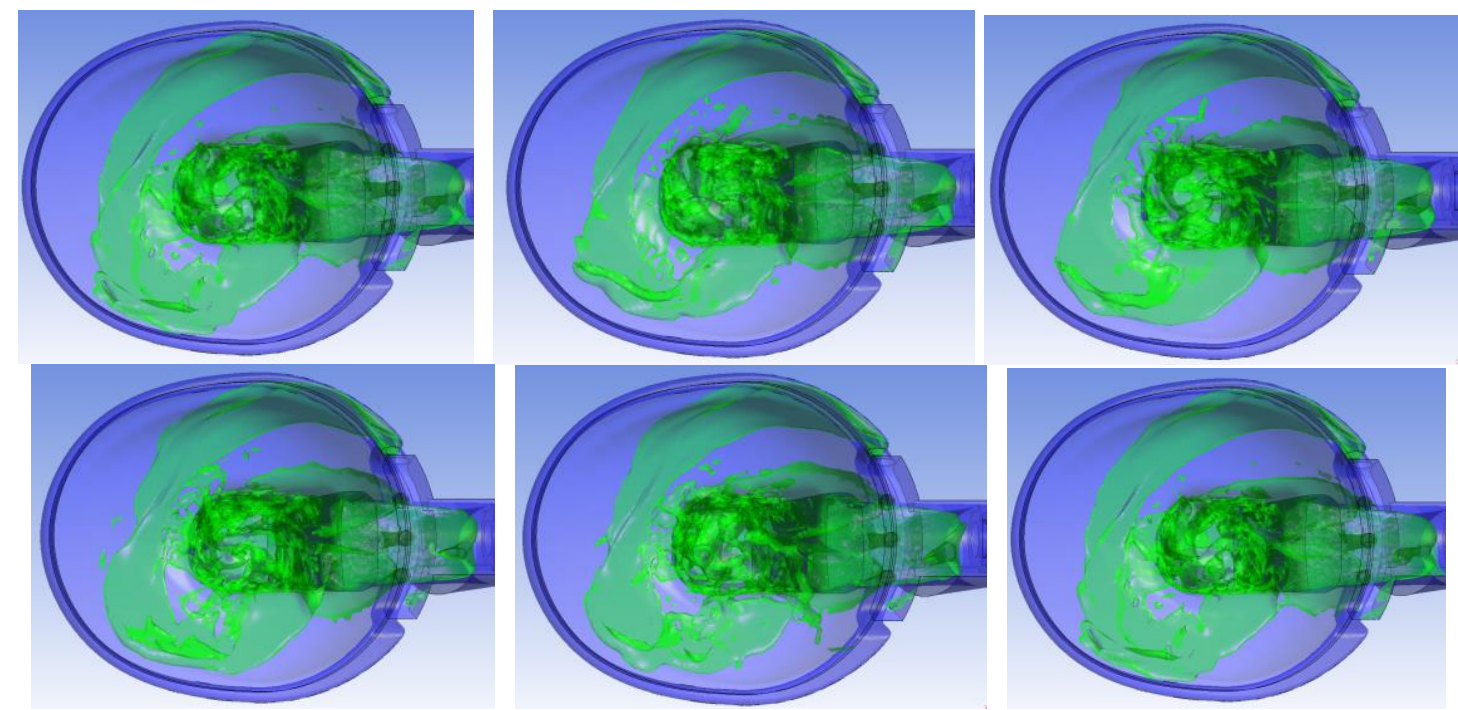

Fig. 6: Free surface visualization for a temporal sequence with a time step of $0.1 \mathrm{~s}$. 
Both experimental and numerical results indicate that no spreading of the discharged water out of the top of the toilet bowl exists. The good agreement between the simulations and the experiments is also a good indicator of the accomplishment of this requirement for the water flow discharge in the toilet bowl.

From the previous work, and given the good agreement between experimental and numerical results, confidence exists on the simulation results of the flow discharge in the toilet bowl. Thus, the numerical approach can be used alone for quick analysis of the water flow discharge and behaviour of different toilet bowls, as well as to analyse the effects of different discharge conditions into the bowl.

\section{Results and Validation}

Once confident on the numerical simulation results, simulations can be made for different designs of the toilet bowl, in order to numerically assess its performance before materialization of the concept under analysis. A new toiled bowl design was considered, which is presented in Fig. 7. This design aimed at a better distribution of water over the bowl surface, a feature that was successfully achieved, as may be appreciated in this figure. The good simulation results led to the development of the toilet bowl prototype, which was experimentally tested. Once again, numerical results agree quite well with the experimental visualisations. In this representation, the water free surface has been coloured with the free surface velocity, thus allowing a better understanding of how the water cleaning capability is distributed over the toilet bowl inner surface.

It must be noted that these results were obtained with an inlet flow rate of $0.58 \mathrm{~L} / \mathrm{s}$, which is lower than the one used in the first design $(0.85 \mathrm{~L} / \mathrm{s})$. This is an important feature in what concerns water saving requirements.
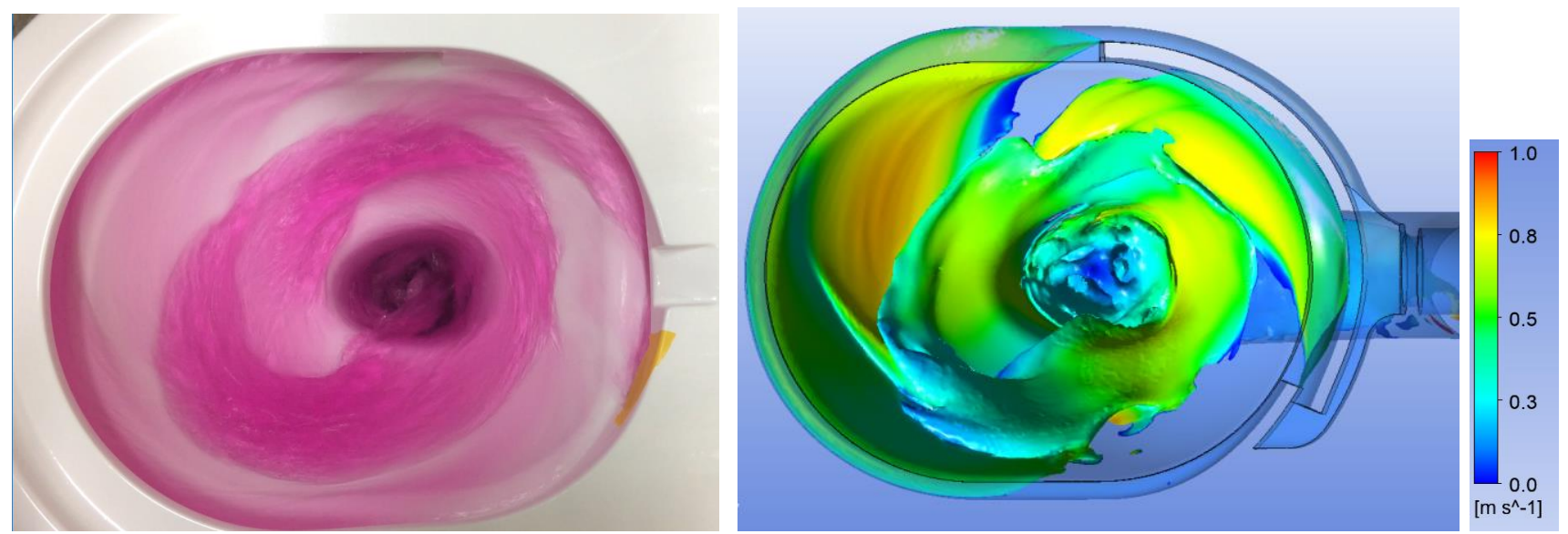

Fig. 7: Experimental (left) and the corresponding numerical (right) results of the water discharge in the new design toilet bowl.

\section{Conclusions}

Numerical simulation can help the development of new products and solutions. If this is true in general, it is also true for the case of toilet bowls, which need to be more and more considered given the demands on design, compactness, water efficiency, and functionality. The main objective is to have compact toilet bowls with attractive design, which are affective for the function but efficient in terms of water consumption. Solutions to market need to be proposed in very short times, requiring short time and low cost development methods.

Experimental results of the discharge process in an existing bowl were used to validate the numerical simulations. Some tuning was made concerning the parameters and approaches selected to conduct the numerical simulations. Once the experimental and numerical results showed a good agreement, confidence exists in the numerical simulations, and further faster and cheaper developments can be made based primarily, or even exclusively, on numerical simulations. This is the case for a new proposed toilet bowl, for which the numerical simulations of the discharge process are presented. The new toilet bowl demonstrated to be a successful design, with a more uniform water distribution associated with a lower discharge rate. 


\section{Acknowledgements}

The present work was conducted under the scope of the project WashOne, Sistema de Incentivos à I\&D Empresarial (Copromoção), 017461.

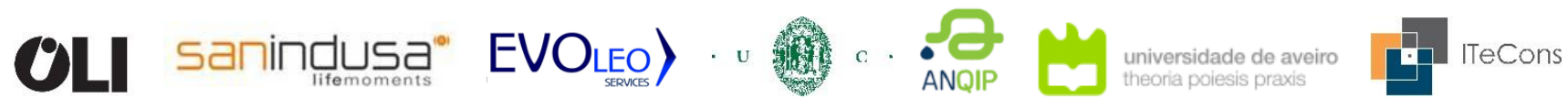

\section{References}

[1] Cuvettes de WC à alimentation indépendante et cuvettes de WC à réservoir attenant (WC pans and WC suites Connecting dimensions), NF EN 33, October 2011.

[2] Cuvettes de WC et cuvettes à réservoir attenant à siphon integre (WC pans and WC suites with integral trap), NF EN 997, May 2012.

[3] Flow Science, Flushing. [Online]. Available: https://www.flow3d.com/industries/manufacturing/flushing/

[4] M.1 Francisco, A. Gameiro Lopes, V. Costa, "Flush with Success. A toilet discharge valve is optimized to reduce household water consumption and maintain performance," ANSYS Advantage, vol. 2, no. 2, [Online]. Available: http://www.ansys.com/-/media/ansys/corporate/resourcelibrary/article/aa-v2-i2-flush-with-success.pdf

[5] ANSYS CFX V18.0, User's Manual. 2017. 